\title{
Several results on conjugacy class sizes of some elements of finite groups
}

\author{
YONGCAI REN \\ Sichuan University School of Mathematics \\ No.399 Wangjiang Road, Chengdu People's \\ REPUBLIC OF CHINA
}

\begin{abstract}
Let $G$ be a finite group. For an element $x$ of $G, x^{G}$ denotes the conjugacy class of $x$ in $G$. $\left|x^{G}\right|$ is called the size of the conjugacy class $x^{G}$. In this paper, we establish several results on conjugacy class sizes of some elements of finite groups. In addition, we give a simple and clearer proof of a known result.
\end{abstract}

Key-Words: Conjugacy class sizes; Finite groups; Conjugacy class size; Primary element; Quasi-Frobenius group.

Received: March 28, 2021. Revised: July 14, 2021. Accepted: July 28, 2021. Published: August 4, 2021.

\section{Introduction and Preliminaries}

Throughout this paper, the term group always means a group of finite order. The letter $G$ always denotes a group, and the letter $p$ always denotes a prime. For an element $x \in G, o(x)$ denotes the order of $x$, and $x^{G}$ denotes the conjugacy class of $x$ in $G$. $\left|x^{G}\right|$ is called the size of the conjugacy class $x^{G}$. We denote by $\pi(G)$ the set of prime divisors of the order $|G|$ of a group $G$. For a positive integer $m, \pi(m)$ denotes the set of prime divisors of $m$. All further unexplained notations are standard and can be found in [1].

Let $x$ be an element of $G$. We say that $x$ is primary if $o(x)$ is a power of a prime (including the case where $x=1$, the identity element); We say that $x$ is biprimary if $o(x)$ is divisible by precisely two distinct primes.

We write

$$
\begin{gathered}
c s(G)=\left\{\left|x^{G}\right|: x \in G\right\}, \\
p c s(G)=\left\{\left|x^{G}\right|: x \in G \text { and } x \text { is primary }\right\}
\end{gathered}
$$

and

$$
\begin{aligned}
& \operatorname{ppcs}(G)=\left\{\left|x^{G}\right|: x \in G \text { and } x\right. \text { is primary or } \\
& \text { biprimary }\} \text {. }
\end{aligned}
$$

We say that $G$ is a quasi-Frobenius group if $G / Z(G)$ is a Frobenius group. The inverse images in $G$ of the kernel and a complement of $G / Z(G)$ are called the kernel and a complement of $G$. It is well-known that if $G$ is a quasi-Frobenius group with abelian kernel and complement, then $\operatorname{cs}(G)=$ $c s(G / Z(G))$ (see [2]).
A classical topic of finite group theory is to study the influence of the conjugacy class sizes on the structure of groups. However, studying such properties only from partial information, provided by connjugacy class sizes of certain elements, can be a more complex and more interesting problem. In this paper we study the influence of the conjugacy class sizes of primary and biprimary elements on the structure of groups. In addition, we give a simple and clearer proof of a known result.

In this section we list some lemmas which will be used in the sequel. The following Lemma 1.1 is well-known.

Lemma 1.1 Let $x \in G$. Assume that $o(x)=$ $p_{1}^{m_{1}} \ldots p_{n}^{m_{n}}$, where $p_{1}, \ldots, p_{n}$ are distinct primes and $m_{1}, \ldots, m_{n}$ are positive integers. Then, $x=x_{1} \ldots x_{n}$ with $o\left(x_{i}\right)=p_{i}^{m_{i}}$ and $x_{r} x_{s}=x_{s} x_{r}$ for $s, r=1, \ldots, n$. Furthermore, there exist integers $k_{i}$ such that $x^{k_{i}}=x_{i}$ for $i=1, \cdots, n$.

Lemma 1.2 ([3, Lemma 2.4]) A prime p does not divide the conjugacy class sizes of primary elements of $G$ if and only if $G$ has a central Sylow p-subgroup.

Lemma 1.3 ([4, LEMMA 1(1)]) Let $a, b \in G$. If $\left(\left|a^{G}\right|,\left|b^{G}\right|\right)=1$, then $G=C_{G}(a) C_{G}(b)$.

Lemma 1.4 ([5, Theorem 6.4.3]) If $G=A B$, where $A$ and $B$ are two nilpotent subgroups of $G$, then $G$ is solvable.

Lemma 1.5 ([6, Lemma 2.1]) Let $G$ be a $\pi$-separable group with $\pi \subseteq \pi(G)$. Then,

(i) $\left|x^{G}\right|$ is a $\pi^{\prime}$-number for every primary $\pi^{\prime}$-element $x$ if and only if $G=O_{\pi}(G) \times O_{\pi^{\prime}}(G)$. 
(ii) $\left|x^{G}\right|$ is a $\pi$-number for every primary $\pi^{\prime}$-element $x$ if and only if $G$ has an abelian Hall $\pi^{\prime}$-subgroup.

Lemma 1.6 ([2, Proposition 1.4]) Suppose that $G / Z(G) \cong S_{3}$, the symmetric group of degree 3 . Then, up to a central direct factor, $G=T D$, where $T$ is a normal subgroup of order $3, D$ a cyclic 2 -group.

The following Lemma 1.7 is a consequence of [7, Theorem A].

Lemma 1.7 If $\operatorname{ppcs}(G)=\{1, m\}$, then $m=p^{a}$ for some prime $p$ and some positive integer $a$, and $G=P \times A$, where $P$ is a $p$-group and $A$ is an $a$ belian group.

\section{Results and Proofs}

A positive integer $m$ is called a Hall number of a group $G$ if $m|| G \mid$ and $(m,|G| / m)=1$.

Theorem 2.1 Suppose that $G$ satisfies the following two conditions:

(1) $p c s(G)=\left\{1, m_{1}, m_{2}\right\}$ with $\left(m_{1}, m_{2}\right)=1$.

(2) For each $p \in \pi(G)$ and any noncentral $p$-element $x$ of $G, C_{G}(x)=C_{G}(x)_{p} \times C_{G}(x)_{p^{\prime}}$ and $C_{G}(x)_{p^{\prime}}$ is abelian.

Then, the following statements are true:

(I) $G$ is a quasi-Frobenius group with abelian $k$ ernel and complement.

(II) Assume that $G$ has no non-trivial abelian direct factor. Set $\pi:=\pi\left(m_{1}\right)$. If $m_{1}$ is a Hall-number of $G$, then $Z(G)_{\pi}=1$. If $m_{2}$ is a Hall-number of $G$, then $Z(G)_{\pi^{\prime}}=1$. If both $m_{1}$ and $m_{2}$ are Hallnumber of $G$, then $Z(G)=1$.

Proof We write $\pi:=\pi\left(m_{1}\right)$. By hypothesis, we have $\pi\left(m_{1}\right) \cap \pi\left(m_{2}\right)=\emptyset$. If a prime divisor $r$ of $|G|$ does not divide $m_{i}$ for $i=1,2$, then by Lemma 1.2 we know that $G$ has a central Sylow $r$-subgroup, and hence $G$ has a non-trivial abelian direct factor.It is well-known that abelian direct factors are immaterial in this context, and so we can assume that $G$ has no non-trivial abelian direct factor. Therefore, we have $\pi(G)=\pi\left(m_{1}\right) \cup \pi\left(m_{2}\right)$. Let $x, y \in G$ be primary elements such that $\left|x^{G}\right|=m_{1}$ and $\left|y^{G}\right|=m_{2}$. Since $\left(m_{1}, m_{2}\right)=1$, by Lemma 1.3 we have $G=C_{G}(x) C_{G}(y)$. By hypothesis(condition (2)), $C_{G}(x)$ and $C_{G}(y)$ are nilpotent, and so by Lemma 1.4 we conclude that $G$ is solvable. Hence, $G$ has Hall $\pi$-subgroups and Hall $\pi^{\prime}$-subgroups. Notice that any two Hall $\pi\left(\pi^{\prime}\right)$-subgroups of $G$ are conjugate, and any $\pi\left(\pi^{\prime}\right)$-subgroup of $G$ is contained a Hall $\pi\left(\pi^{\prime}\right)$-subgroup of $G$.

Let $z \in G$ be a primary $\pi$-element, and suppose that $\left|z^{G}\right|=m_{1}$. Then, by hypothesis(condition (2)) we have

$$
\text { (*) } C_{G}(z)=C_{G}(z)_{\pi} \times H,
$$

where $H$ is a Hall $\pi^{\prime}$-subgroup of $G$ and $H$ is abelian. Let $K$ be a Hall $\pi$-subgroup of $G$ such that $C_{G}(z)_{\pi} \subseteq K$. Clearly, we have $C_{G}(z)_{\pi}<$ $K$. For every noncentral primary element $x \in$ $C_{G}(z)_{\pi}$, by equality $(*)$ it is obvious that $\left|x^{G}\right|$ is a $\pi$-number.There exists a noncentral primary element $w \in K-C_{G}(z)_{\pi}$ such that $\left|w^{G}\right|$ is a $\pi^{\prime}$-number; otherwise,for every noncentral primary element $y$ of $K$ we have that $\left|y^{G}\right|$ is a $\pi$-number, and thus by Lemma 1.5(i) we have that $G=K \times H$ and the abelian Hall $\pi^{\prime}$-subgroup $H$ of $G$ is a central direct factor of $G$, forcing $\operatorname{pcs}(G)=\left\{1, m_{1}\right\}$, in contradiction to the hypothesis of the theorem. Then, there exists some element $g$ such that $K \leq C_{G}(w)^{g}=C_{G}\left(w^{g}\right)$. It follows that $w^{g} \leq Z(K)$. Then, since $C_{G}(z)_{\pi} \subseteq K$, $w^{g} \in C_{G}(z)_{\pi}$, and thus by equality $(*)$ we have $H \leq C_{G}\left(w^{g}\right)$. It follows that $w \in Z(G)$, contrary to the choice of $w$. Consequently, we conclude that every primary $\pi$-element of $G$ has conjugacy class size 1 or $m_{2}$. Then, by Lemma 1.5(ii) we conclude that Hall $\pi$-subgroups of $G$ are abelian. By the same arguments we conclude that Hall $\pi^{\prime}$-subgroup of $G$ are abelian. Let $K$ be a Hall $\pi$-subgroup of $G$, and let $H$ be a Hall $\pi^{\prime}$-subgroup of $G$. Then, $K$ and $H$ are abelian.

Let $x$ be a noncentral primary $\pi$-element of $G$. We may assume that $x \in K$. Since $K$ is abelian, by hypothesis we have $C_{G}(x)=K \times C_{G}(x)_{\pi^{\prime}}$. We may assume that $C_{G}(x)_{\pi^{\prime}} \leq H$. Then, since $H$ is abelian, we conclude that $C_{G}(x)_{\pi^{\prime}}=Z(G)_{\pi^{\prime}}$. Let $y \in G$ be a noncentral primary $\pi^{\prime}$-element of $G$. By the same arguments as for $x$ we conclude that $C_{G}(y)_{\pi}=Z(G)_{\pi}$.

Since $K$ and $H$ are abelian, we have $F(G)=$ $O_{\pi}(G) \times O_{\pi^{\prime}}(G)$. Since $G$ is nonabelian, by [5, 4.2, p.277] we have $F(G) \not \leq Z(G)$. Then, either $O_{\pi}(G) \not \subset Z(G)$ or $O_{\pi^{\prime}}(G) \not \leq Z(G)$. Without loss of generality, we may assume that $O_{\pi}(G) \not Z Z(G)$. Suppose that $O_{\pi^{\prime}}(G) \not \leq Z(G)$, and let $y \in O_{\pi^{\prime}}(G)-$ $Z(G)$. By the above paragraph we have $C_{G}(y)_{\pi}=$ $Z(G)_{\pi}$. Then, since $O_{\pi}(G) \leq C_{G}\left(O_{\pi^{\prime}}(G)\right) \leq$ $C_{G}(y)$, we have $O_{\pi}(G) \leq Z(G)$, contradicting our assumption. Therefore, we conclude that $O_{\pi^{\prime}}(G)=$ $Z(G)_{\pi^{\prime}}$. It follows that $K \leq C_{G}\left(O_{\pi}(G)\right)=$ $\left.C_{G}\left(O_{\pi}(G)\right) \times Z(G)_{\pi^{\prime}}\right)=C_{G}\left(O_{\pi}(G) \times O_{\pi^{\prime}}(G)\right)=$ $C_{G}(F(G)) \leq F(G)=O_{\pi}(G) \times O_{\pi^{\prime}}(G)($ see $[5,4.2$, p.277]), and thus $K=O_{\pi}(G)$, that is, $K \triangleleft G$. Then, we have $G=K>\triangleleft H$. (The notation $>\triangleleft$ denotes a semidirect product.)

Consider the factor group $G / Z(G)=$ $K H / Z(G)$, and we use the bar convention. Let $\bar{h}$ be a 
primary non-identity element of $\bar{H}=H Z(G) / Z(G)$, and let $\bar{k}$ be a primary non-identity element of $\bar{K}=K Z(G) / Z(G)$. By Lemma 1.1 we may assume that $h$ and $k$ are primary.Suppose that $[\bar{k}, \bar{h}]=\overline{1}$. Then we have that $[k, h] \leq Z(G)$. It follows that $[k, h] \leq[K, H] \cap C_{K}(H)$. On the other hand, we have that $K=[K, H] \times C_{K}(H)$ (see [8, Theorem 2.3 , p.177]). Hence, we get that $[k, h]=1$.It follows that $h \in C_{G}(k)_{\pi^{\prime}}=Z(G)_{\pi^{\prime}}$, and thus $\bar{h}=\overline{1}$, a contradiction. So, we have that $[\bar{k}, \bar{h}] \neq \overline{1}$. Then, by Lemma 1.1 and [9, Problems 7.1(a), p.121] we conclude that $G / Z(G)=K H / Z(G)$ is a Frobenius group with the kernel $K Z(G) / Z(G)$ and a complement $H Z(G) / Z(G)$. Thus, noting that $K$ and $H$ are abelian, $G$ is a quasi-Frobenius group with abelian kernel and complement. So, statement (I) holds.

We have that $m_{1}=\left|K / Z(G)_{\pi}\right|$ and $m_{2}=$ $\left|H / Z(G)_{\pi^{\prime}}\right|$, and so statement (II)) is obvious. This completes the proof of the theorem.

Theorem 2.2 [10, THEOREM 1.1] Suppose that $\operatorname{ppcs}(G)=\left\{1, m_{1}, m_{2}\right\}$ with $\left(m_{1}, m_{2}\right)=1$. Then, $G$ is a quasi-Frobenius group with abelian kernel and complement.

Proof By hypothesis and Lemma 1.2 we have that $p c s(G)=\left\{1, m_{1}, m_{2}\right\}$ with $\left(m_{1}, m_{2}\right)=1$.

For any $p \in \pi(G)$, let $x$ be a $p$-element of $G$ such that $\left|x^{G}\right|=m_{i}$ for $i=1$ or 2 . Let $z$ be any primary $p^{\prime}$-element of $C_{G}(x)$. We have that $C_{G}(x z)=C_{G}(x) \cap C_{G}(z) \leq C_{G}(x)$. Then, since $\left(m_{1}, m_{2}\right)=1$, we conclude that $C_{G}(x)=$ $C_{G}(x z) \leq C_{G}(z)$, and thus $z \in Z\left(C_{G}(x)\right)$. Hence, $C_{G}(x)=C_{G}(x)_{p} \times C_{G}(x)_{p^{\prime}}$ and $C_{G}(x)_{p^{\prime}}$ is abelian.

To sum up, $G$ satisfies two conditions of Theorem 2.1. Hence, by Theorem 2.1 we conclude that the theorem holds. This completes the proof.

Note In the original proof of [10, THEOREM 1.1], the authors of [10] dealt separately with the following three cases: (i) Both $m_{1}$ and $m_{2}$ are Hall numbers of $G$;(ii) Only one of $m_{1}$ and $m_{2}$ is a Hall number of $G$; (iii) Neither $m_{1}$ nor $m_{2}$ is a Hall number of $G$. From the proof of Theorem 2.1, we see that it is not necessary to deal separately with the above-mentioned three cases. So, we have given a simple and clearer proof of [10, THEOREM 1.1]. However, we point out that, in fact, THEOREM 1.1 of [10] is a consequence of Theorem 1 and Theorem 2 of [4] (see the following Theorem 2.5 and its proof). In addition, we will see that THEOREM 1.1 of [10] is also a consequence of Corollary 2.12 in the present paper.

Corollary 2.3 Suppose that $\operatorname{ppcs}(G)=$ $\{1, n, n+1\}$. Then, $G$ is a quasi-Frobenius group with abelian kernel and complement. Furthermore, $n+1$ is a prime power.

Proof Clearly, $(n, n+1)=1$. Then, by Theorem 2.2, $G / Z(G)=K / Z(G)>\triangleleft H / Z(G)$ is a Frobenius group with the kernel $K / Z(G)$ and a complement $H / Z(G)$, and $K$ and $H$ are abelian. Since $\{1,|H / Z(G)|,|K / Z(G)|\}=\operatorname{cs}(G / Z(G))=$ $\operatorname{cs}(G)$, we conclude that $\{1,|H / Z(G)|,|K / Z(G)|\}=$ $\{1, n, n+1\}$.Hence, $G / Z(G)$ is a sharply 2 -transitive group of degree $n+1$, and so by [11, XII Theorem 9.1] we conclude that $n+1$ is a prime power. This completes the proof.

Corollary 2.4 Suppose that $\operatorname{ppcs}(G)=$ $\{1,2,3\}$. Then, $G / Z(G) \cong S_{3}$. Furthermore, up to a central direct factor, $G=D T$, where $D$ is a normal subgroup of order 3 and $T$ is a cyclic 2-group.

Proof By Corollary 2.3 and its proof we conclude that $G / Z(G)$ is a nonabelian group of order 6 , and so $G / Z(G) \cong S_{3}$. Thus, by Lemma 1.6 we get that, up to an abelian direct factor, $G=D T$, where $D$ is a normal subgroup of order 3 and $T$ is a cyclic 2 -group. This completes the proof.

\section{Theorem 2.5 Suppose that}

$$
\operatorname{ppcs}(G)=\left\{1, m_{1}, \cdots, m_{t}\right\}
$$

with $t \geq 2$.In addition, for $i, j=1, \cdots, t$, if $i \neq j$, then $\left(m_{i}, m_{j}\right)=1$.Then, $t=2$ and $G$ is a quasiFrobenius group with abelian kernel and complement.

Proof Let $x$ be a noncentral element of $G$, and assume that $o(x)=p_{1}^{r_{1}} \ldots p_{n}^{r_{n}}$, where $p_{1}, \ldots, p_{n}$ are distinct primes and $r_{1}, \ldots, r_{n}$ are positive integers. By Lemma 1.1 we have that $x=x_{1} \ldots x_{n}$ with $o\left(x_{i}\right)=p_{i}^{r_{i}}$ and $x_{r} x_{s}=x_{s} x_{r}$ for $s, r=1, \ldots, n$. Since $x$ is noncentral, some $x_{i}$ is noncentral for $1 \leq i \leq n$. Without loss of generality, we may assume that $x_{1}$ is noncentral. By hypothesis, we have $\left|x_{1}^{G}\right|=m_{j}$ for some $j$.Clearly, we may assume that $j=1$, that is, $\left|x_{1}^{G}\right|=m_{1}$. By the same arguments as in the proof of Theorem 2.2 we conclude that $x_{i} \in Z\left(C_{G}\left(x_{1}\right)\right)$ for $i=1, \cdots, n$. It follows that $C_{G}\left(x_{1}\right) \subseteq C_{G}\left(x_{i}\right)$ for $i=2, \cdots, n$.Thus,

$$
\begin{gathered}
C_{G}(x)=C_{G}\left(x_{1} \cdots x_{n}\right)= \\
C_{G}\left(x_{1}\right) \cap C_{G}\left(x_{2}\right) \cap \cdots \cap C_{G}\left(x_{n}\right)=C_{G}\left(x_{1}\right) .
\end{gathered}
$$

It follows that $\left|x^{G}\right|=\left|x_{1}^{G}\right|=m_{1}$. So, we have proved that $c s(G)=\operatorname{ppcs}(G)=\left\{1, m_{1}, \cdots, m_{t}\right\}$. Then, since $\left(m_{i}, m_{j}\right)=1$ for $i \neq j$, by THEOREM 1 of [4] we conclude that $t=2$, and so by THEOREM 2 of [4] (or by Theorem 2.2 in the precent paper) we conclude that $G$ is a quasi-Frobenus group with abelian kernel and complement. This completes the proof.

The following Theorem 2.6 is an extension of [4, COROLLARY 3]. 
Theorem 2.6 Let $G$ be a nonabelian group, and suppose that $G$ satisfies the following condition:

(*) Let $x, y \in G-Z(G)$ be primary or biprimary. If $x^{G} \neq y^{G}$, then $\left(\left|x^{G}\right|,\left|y^{G}\right|\right)=1$.

Then, $G \cong S_{3}$.

Proof In view of condition $(*)$, by Theorem 2.5 and Lemma 1.7, one of the following two cases may occur:

(1) $G=P \times A$, where $P$ is a $p$-group for some prime $p$ and $A$ is an abelian group.Furthermore, if $x, y \in G-Z(G)$ are primary or biprimary, then $x^{G}=y^{G}$.

In this case, it is clear that $A=1$ and $G$ is a $p$-group. Furthermore, $G$ has only one conjugacy class of noncentral elements. It follows that $|G|=$ $|Z(G)|+|G| / n$, where $n$ is a positive integer and $n \geq 2$. Then, $|Z(G)| \geq|G| / 2$, and this implies that $G$ is abelian, a contradiction because $G$ is nonabelian by hypothesis. So, this case can not occur.

(2) $G$ is a quasi-Frobenius group. Furthermore, $G$ has exactly two classes of noncentral primary and biprimary elements.

In this case, it is easy to see that $|\pi(G)|=2$ and $Z(G)=1$. Then, $G=P>\triangleleft Q$ is a Frobenius group with the kernel $P$ and a complement $Q$, where $P$ is an abelian $p$-group and $Q$ is an abelian $q$-group, $p$ and $q$ are two distinct primes. Suppose that $|Q|>2$. Then, there exist non-identity elements $x, y \in Q$ such that $x \neq y$. We have $x^{G}=y^{G}$, and thus there exists a non-identity element $w \in P$ such that $x^{w}=y$. It follows that $y \in Q^{w} \cap Q=1$ (see [5, 8.5, p.497]), and so $y=1$, a contradiction. Hence, we have $|Q|=2$. Then, we have $|P|-1=2$, and thus $|P|=3$ and $G \cong S_{3}$. The proof is complete.

\section{Theorem 2.7 Suppose that}

$$
\operatorname{ppcs}(G)=\{1, n, n+1, \cdots, n+r\}
$$

with $r \geq 1$. If ppcs $(G)$ does not contain any prime, then $r=1$ and $G$ is a quasi-Frobenius group with abelian kernel and complement. Furthermore, $n+1$ is a prime power.

Proof Let $x$ be a noncentral element of $G$. We have that that $o(x)=p_{1}^{m_{1}} \ldots p_{k}^{m_{k}}$, where $p_{1}, \ldots, p_{k}$ are distinct primes and $m_{1}, \ldots, m_{k}$ are positive integers. By Lemma 1.1 we have that $x=x_{1} \ldots x_{k}$ with $o\left(x_{i}\right)=p_{i}^{m_{i}}$ and $x_{t} x_{s}=x_{s} x_{t}$ for $s, t=$ $1, \ldots, k$. Since $x$ is noncentral, some $x_{i}$ is noncentral for $1 \leq i \leq k$. Without loss of generality, we may assume that $x_{1}$ is noncentral. For $i \neq 1$, we have $C_{G}\left(x_{1} x_{i}\right)=C_{G}\left(x_{1}\right) \cap C_{G}\left(x_{i}\right)$, and so $C_{G}\left(x_{1} x_{i}\right) \leq C_{G}\left(x_{1}\right)$. If $C_{G}\left(x_{1} x_{i}\right)<C_{G}\left(x_{1}\right)$, then $\left|x_{1}^{G}\right|$ is a proper divisor of $\left|\left(x_{1} x_{i}\right)^{G}\right|$, so that $2\left|x_{1}^{G}\right| \leq\left|\left(x_{1} x_{i}\right)^{G}\right|$. By the Bertrand's postulate, there exists a prime $p$ such that $\left|x_{1}^{G}\right|<p<2\left|x_{1}^{G}\right| \leq$ $\left|\left(x_{1} x_{i}\right)^{G}\right|$. Since $\left\{\left|x_{1}^{G}\right|,\left|\left(x_{1} x_{i}\right)^{G}\right|\right\} \subseteq \operatorname{ppcs}(G)$, we get that $p \in \operatorname{ppcs}(G)$, contradicting the assumption of the theorem. Hence, $C_{G}\left(x_{1}\right)=C_{G}\left(x_{1} x_{i}\right)=$ $C_{G}\left(x_{1}\right) \cap C_{G}\left(x_{i}\right)$. It follows that $C_{G}\left(x_{1}\right) \leq C_{G}\left(x_{i}\right)$ for $i=2, \cdots, k$. Hence, we have

$$
\begin{gathered}
C_{G}(x)=C_{G}\left(x_{1} \cdots x_{k}\right)= \\
C_{G}\left(x_{1}\right) \cap C_{G}\left(x_{2}\right) \cap \cdots \cap C_{G}\left(x_{k}\right)=C_{G}\left(x_{1}\right) .
\end{gathered}
$$

It follows that $\left|x^{G}\right|=\left|x_{1}^{G}\right| \in \operatorname{ppcs}(G)$. So, we have proved that

$$
c s(G)=\operatorname{ppcs}(G)=\{1, n, n+1, \cdots, n+r\} .
$$

Then, by [2, Theorem 2 ] we conclude that the theorem holds. This completes the proof.

The following Theorem 2.8 is an extension of [12, Theorem 1].

Theorem 2.8 Suppose that $G$ satisfies the following two conditions:

(1) For $r, t \in \operatorname{ppcs}(G)-\{1\}$, if $r \neq t$, then $r \quad \not t$ and $t X_{r}$

(2) $|\operatorname{ppcs}(G)-\{1\}| \geq 3$.

Then, for any three distinct numbers $r_{1}, r_{2}$ and $r_{3}$ in $\operatorname{ppcs}(G)-\{1\}$, if $\left(r_{1}, r_{2}\right)=1$, then $\left(r_{1}, r_{3}\right) \neq 1$ and $\left(r_{2}, r_{3}\right) \neq 1$.

Proof It is easy to show that $p c s(G)=p p c s(G)$. Let $x \in G$ be a noncentral $p$-element for some prime $p$, and let $y \in C_{G}(x)$ be a primary $p^{\prime}-$ element. We have that $C_{G}(x y)=C_{G}(x) \cap C_{G}(y) \leq C_{G}(x)$. By condition (1) we conclude that $C_{G}(x)=C_{G}(x y) \leq$ $C_{G}(y)$, and so $y \in Z\left(C_{G}(x)\right)$. It follows that $C_{G}(x)=C_{G}(x)_{p} \times C_{G}(x)_{p^{\prime}}$ and $C_{G}(x)_{p^{\prime}}$ is abelian. Suppose that $C_{G}(x)_{p^{\prime}} \not \leq Z(G)$. Let $u \in$ $C_{G}(x)_{p^{\prime}}-Z(G)$ be a $q$-element for some prime $q$. We have $q \neq p$. Reapeating the previous arguments, we conclude that $C_{G}(u)=C_{G}(u)_{q} \times C_{G}(u)_{q^{\prime}}$ and $C_{G}(u)_{q^{\prime}}$ is abelian. Then, since $C_{G}(x)_{p} \leq C_{G}(u)_{q^{\prime}}$, $C_{G}(x)_{p}$ is abelian. It follows that $C_{G}(x)$ is abelian. So, we have proved that if $x$ is a noncentral primary element of $G$,then (i) $C_{G}(x)$ is abelian, or (ii) $C_{G}(x)=C_{G}(x)_{p} \times Z(G)_{p^{\prime}}$ for some prime $p$.

Suppose that $\left(r_{1}, r_{3}\right)=1$. Let $x_{1}, x_{2}$ and $x_{3}$ be three noncentral primary elements of $G$ such that $\left|x_{i}^{G}\right|=r_{i}$ for $i=1,2,3$. We claim that $C_{G}\left(x_{1}\right)$ is abelian. Suppose on the contrary that $C_{G}\left(x_{1}\right)$ is not abelian. By the above paragraph we have that $C_{G}\left(x_{1}\right)=C_{G}\left(x_{1}\right)_{p} \times Z(G)_{p^{\prime}}$ for some prime $p$. Then, we have

$$
\begin{gathered}
|G|=\left|x_{1}^{G}\right|\left|C_{G}\left(x_{1}\right)\right|=r_{1}\left|C_{G}\left(x_{1}\right)_{p}\right| Z(G)_{p^{\prime}} \mid= \\
r_{1} p^{a}\left|Z(G)_{p^{\prime}}\right|,
\end{gathered}
$$


where $a$ is some positive integer. It follows that $|G: Z(G)| \mid r_{1} p^{a}$. Then, noting that $r_{2}|| G / Z(G) \mid$ and $r_{3}|| G / Z(G) \mid$, we get that $r_{2} \mid r_{1} p^{a}$ and $r_{3} \mid r_{1} p^{a}$. Then, since $\left(r_{1}, r_{2}\right)=\left(r_{1}, r_{3}\right)=1$, we conclude that $r_{2} \mid p^{a}$ and $r_{3} \mid p^{a}$, and thus either $r_{2} \mid r_{3}$ or $r_{3} \mid r_{2}$, against condition (1).Hence, we have proved that $C_{G}\left(x_{1}\right)$ is abelian. Suppose that $C_{G}\left(x_{2}\right)$ is not abelian. By using the arguments used for $C_{G}\left(x_{1}\right)$ we conclude that $\mid G: Z(G) \| r_{2} q^{b}$, where $q$ is some prime and $b$ is some positive integer.Then, since $r_{1}\|G: Z(G)\| r_{2} q^{b}$ and $\left(r_{1}, r_{2}\right)=1$, we conclude that $r_{1}$ is a power of $q$. Since $\left(r_{1}, r_{3}\right)=1$, we have $q / \mid r_{3}$. Then, since $r_{3}\|G: Z(G)\| r_{2} q^{b}$ we get that $r_{3} \mid r_{2}$, against condition (1). Hence, $C_{G}\left(x_{2}\right)$ is also abelian.

Since $\left(r_{1}, r_{2}\right)=1$, by Lemma 1.3 we have $G=$ $C_{G}\left(x_{1}\right) C_{G}\left(x_{2}\right)$. Then, since $C_{G}\left(x_{1}\right)$ and $C_{G}\left(x_{2}\right)$ are abelian, we have that $C_{G}\left(x_{1}\right) \cap C_{G}\left(x_{2}\right)=Z(G)$. It follows that

$$
\begin{gathered}
|G: Z(G)|=\left|G: C_{G}\left(x_{1}\right) \cap C_{G}\left(x_{2}\right)\right|= \\
|G|\left|C_{G}\left(x_{1}\right) C_{G}\left(x_{2}\right)\right| /\left|C_{G}\left(x_{1}\right)\right|\left|C_{G}\left(x_{2}\right)\right|, \\
|G: Z(G)|=|G|^{2} /\left|C_{G}\left(x_{1}\right)\right|\left|C_{G}\left(x_{2}\right)\right|= \\
\left|x_{1}^{G}\right|\left|x_{2}^{G}\right|=r_{1} r_{2} .
\end{gathered}
$$

Then, $r_{3} \mid r_{1} r_{2}$. Thus, since $\left(r_{1}, r_{3}\right)=1$, we get that $r_{3} \mid r_{2}$, against condition (1). Hence, we have $\left(r_{1}, r_{3}\right) \neq 1$.

By symmetry, we have $\left(r_{2}, r_{3}\right) \neq 1$. This completes the proof of the theorem.

Corollary 2.9 Suppose that ppcs $(G)=\{1,2 k+$ $1,2 k+3, \cdots, 2 l+1\}$ (continuous odd numbers) with $l \leq 3 k$. Then, $p p c s(G)=\{1,2 k+1\}, 2 k+1=p^{a}$ for some prime $p$, and $G=P \times A$, where $P$ is a $p$-group and $A$ is an abelian group.

Proof Since $l \leq 3 k$ by hypothesis, $G$ satisfies condition (1) of Theorem 2.8. Since $(2 k+1,2 k+$ $3)=1$ and $(2 k+1,2 k+5)=1$, by Theorem 2.8 we conclude that $|\operatorname{ppcs}(G)-\{1\}| \leq 2$.

(i) Assume that $|\operatorname{ppcs}(G)-\{1\}|=2$.

In this case, $\operatorname{ppcs}(G)=\{1,2 k+1,2 k+3\}$. By Theorem 2.2, $G / Z(G)=K H / Z(G)$ is a Frobenius group with the kernel $K / Z(G)$ and a complement $H / Z(G)$, and $K$ and $H$ are abelian. Then,we have $c s(G)=\{1,|H / Z(G)|,|K / Z(G)|\}$. It follows that $2 k+3=|K / Z(G)|$ and $2 k+1=|H / Z(G)|$. Then, $2 k+1=|H / Z(G)||| K / Z(G) \mid-1=2 k+2($ see $[5$, 8.3, p.497]). But, this is impossible.

(ii) Assume that $|\operatorname{ppcs}(G)-\{1\}|=1$.

In this case, $\operatorname{ppcs}(G)=\{1,2 k+1\}$. Then, by Lemma 1.7 we conclude that $2 k+1=p^{a}$ for some prime $p$, and $G=P \times A$, where $P$ is a $p$-group and $A$ is an abelian group. This completes the proof of theorem.
We recall that a nonabelian group $G$ is an $\mathbf{F}$-group if the centralizers of its noncentral elements are pairwise incomparable with respect to inclusion, that is, for $x, y \in G-Z(G)$, we have that $C_{G}(x) \leq C_{G}(y)$ implies that $C_{G}(x)=C_{G}(y)$.

We have the following

Theorem A [13, Rebmann] A nonabelian solvable group $G$ is an $\mathbf{F}$-group if and only if it is one of the following types:

(i) $G=P \times A$, where $P$ is an $\mathbf{F}$-group of prime power order and $A$ is abelian; index;

(ii) $G$ has an abelian normal subgroup of prime

(iii) $G$ is a quasi-Frobenius group with abelian kernel and complement;

(iv) $G / Z(G)$ is a Frobenius group with the kernel $K / Z(G)$ and a complement $L / Z(G)$, where $L$ is abelian, $Z(K)=Z(G), K / Z(G)$ has prime power order and $K$ is an $\mathbf{F}$-group.

(v) $G / Z(G) \cong S_{4}$, and $V$ is not abelian if $V / Z(G)$ is the Klein four group in $G / Z(G)$.

Theorem 2.10 Let $G$ be a nonabelian group. Suppose that for noncentral primary or biprimary elements $x, y$ of $G, C_{G}(x) \leq C_{G}(y)$ implies $C_{G}(x)=C_{G}(y)$. Then, $c s(G)=p c s(G)=p p c s(G)$ and $G$ is an $\mathbf{F}$-group.

Proof Let $z$ be any noncentral element of $G$.We have that $o(z)=p_{1}^{m_{1}} \ldots p_{n}^{m_{n}}$, where $p_{1}, \ldots, p_{n}$ are distinct primes and $m_{1}, \ldots, m_{n}$ are positive integers. By Lemma 1.1 we have that $z=z_{1} \ldots z_{n}$ with $o\left(z_{i}\right)=p_{i}^{m_{i}}$ and $z_{r} z_{s}=z_{s} z_{r}$ for $s, r=1, \ldots, n$. Since $z$ is noncentral, some $z_{i}$ is noncentral. Without loss of generality we may assume that $z_{1}$ is noncentral. For $i=2, \cdots, n$, we have that $C_{G}\left(z_{1} z_{i}\right)=C_{G}\left(z_{1}\right) \cap$ $C_{G}\left(z_{i}\right) \leq C_{G}\left(z_{1}\right)$. Then, by hypothesis we have $C_{G}\left(z_{1} z_{i}\right)=C_{G}\left(z_{1}\right)$. On the other hand, we have $C_{G}\left(z_{1} z_{i}\right)=C_{G}\left(z_{1}\right) \cap C_{G}\left(z_{i}\right) \leq C_{G}\left(z_{i}\right)$. It follows that $C_{G}\left(z_{1}\right) \leq C_{G}\left(z_{i}\right)$ for $i=2, \cdots, n$. Hence, we have

$$
\begin{gathered}
C_{G}(z)=C_{G}\left(z_{1} \cdots z_{n}\right)=C_{G}\left(z_{1}\right) \cap C_{G}\left(z_{2} \cdots z_{n}\right)= \\
C_{G}\left(z_{1}\right) \cap C_{G}\left(z_{2}\right) \cap \cdots \cap C_{G}\left(z_{n}\right)=C_{G}\left(z_{1}\right) .
\end{gathered}
$$

So, we have $C_{G}(z)=C_{G}\left(z_{1}\right)$ and we have proved that $c s(G)=p c s(G)=p p c s(G)$.

Let $x, y$ be noncentral elements of $G$ such that $C_{G}(x) \leq C_{G}(y)$. By the above paragraph, there exist noncentral primary elements $x_{1}$ and $y_{1}$ of $G$ such that $C_{G}(x)=C_{G}\left(x_{1}\right)$ and $C_{G}(y)=C_{G}\left(y_{1}\right)$. Then, $C_{G}\left(x_{1}\right) \leq C_{G}\left(y_{1}\right)$. By hypothesis, we have $C_{G}\left(x_{1}\right)=C_{G}\left(y_{1}\right)$. It follows that $C_{G}(x)=C_{G}(y)$. So, we have proved that, for any noncentral elements $x, y$ of $G, C_{G}(x) \leq C_{G}(y)$ implies $C_{G}(x)=C_{G}(y)$. 
Hence, $G$ is an $\mathbf{F}$-group. This completes the proof of the theorem.

Theorem 2.11 Let $G$ be a nonabelian group, and suppose that ppcs $(G)$ satisfies the following condition: For $r, t \in \operatorname{ppcs}(G)$, if $r \neq t$, then $r \Lambda t$ and $t$ Xr. Then, the following two propositions hold:

(1) $c s(G)=p c s(G)=p p c s(G)$;

(2) If $G$ is solvable, then $G$ is one of the following types:

(i) $G=P \times A$, where $P$ is a $p$-group for some prime $p$ and $A$ is an abelian group. Further, $\operatorname{ppcs}(G)=c s(P)=\left\{1, p^{a}\right\}$, where a is a fixed positive integer;

(ii) $G$ is a quasi-Frobenius group with abelian $k$ ernel and complement;

(iii) $G=P L$, where $P$ is a normal Sylow $p$-subgroup of $G$ for some prime $p$ and $L$ is an $a$ belian p-complement of $G$, and $G / Z(G)$ is a Frobenius group. Furthermore, $Z(P)=Z(G) \cap P$ and $|c s(P)|=2$.

Proof By hypothesis and Theorem 2.10 we conclude that $c s(G)=\operatorname{ppcs}(G)=p c s(G)$ and $G 1 \mathrm{~s}$ an F-group. Thus, proposition (1) holds.

Now, we prove proposition (2). By the assumption of (2), $G$ is solvable. Then, $G$ is a solvable $\mathbf{F}$ group. Therefore, by Theorem A, we have the following types of groups:

(a) $G=P \times A$,where $P$ is an $\mathbf{F}$-group of prime power order and $A$ is abelian.

In this case, $P$ is a $p$-group for some prime $p$. We can assume that $A=1$. Then $G=P$, and by hypothesis we have $\operatorname{ppcs}(G)=c s(G)=c s(P)=$ $\left\{1, p^{a}\right\}$, where $a$ is a fixed positive integer. So, $G$ is of type (i).

(b) $G$ is nonabelian and has an abelian normal subgroup of prime index $p$.

If $G$ is nilpotent, then it is easy to see that $G$ is of type (i). So, we may assume that $G$ is not nilpotent. Then, it is clear that $G=K P$, where $P$ is a Sylow $p$-subgroup of $G$ and $K$ is an abelian normal $p$-complement of $G$, and $\left|P / O_{p}(G)\right|=p$.

Since $K$ is abelian normal and $(|K|,|P|)=1$, we have $K=[K, P] \times C_{K}(P)$. Clearly, $C_{K}(P)$ is an abelian direcy factor of $G$. We can assume that $G$ has no nontrivial abelian direct factor, and so $C_{K}(P)=1$ and $K=[K, P]$. Then, it is obvious that $G / O_{p}(G)$ is a Frobenius group with the kernel $K O_{p}(G) / O_{p}(G) \cong K$ and a complement $P / O_{p}(G)$ of order $p$. Suppose that $P$ is nonabelian. Then, it is easy to see that $c s(G)=\left\{1, p, p^{a}|K|\right\}$ for some positive integer $a$ (see also [14, Lemma 3.3]).Thus, since $\operatorname{ppcs}(G)=c s(G)($ see $(1)), \operatorname{ppcs}(G)$ does not satisfy the condition of the theorem, a contradiction. Therefore, $P$ is abelian, $O_{p}(G)=Z(G)$ as $C_{K}(P)=1$, and $G / Z(G)=K P / Z(G)$ is a Frobenius group. Then, $G$ is of type (ii).

(c) $G$ is a quasi-Frobenius group with abelian kernel and complement.

This is of type (ii).

(d) $G / Z(G)$ is a Frobenius group with the kernel $K_{0} / Z(G)$ and a complement $L_{0} / Z(G)$, where $L_{0}$ is abelian, $Z\left(K_{0}\right)=Z(G), K_{0} / Z(G)$ has prime power order and $K_{0}$ is an $\mathbf{F}$-group.

In this case, $K_{0} / Z(G)$ is a $p$-group for some prime $p$. Then, $K_{0}=P \times A$, where $P$ is a Sylow $p$-subgroup of $K$, and $A$ is an abelian group.Clearly, $P \triangleleft G$ and $Z(G)=Z\left(K_{0}\right)=Z(P) \times A$. Further, since $K_{0}$ is an $\mathbf{F}$-group and $A$ is abelian, we have $|c s(P)|=2$. We have

$$
\begin{gathered}
G / Z(G)=K_{0} / Z(G)>\triangleleft L_{0} / Z(G) \\
\cong P / Z(P)>\triangleleft L_{0} / Z(G) .
\end{gathered}
$$

Then, since $G / Z(G)$ is a Frobenius group with the kernel $K_{0} / Z(G)$ and a complement $L_{0} / Z(G)$, we have $p \bigwedge\left|L_{0} / Z(G)\right|$. Let $L$ be the $p$-complement of $L_{0}$. Since $L_{0}$ is abelian, $L$ is abelian. So, we have that $G=P L$ and $G$ is of type (iii).

(e) $G / Z(G) \cong S_{4}$, and $V$ is not abelian if $V / Z(G)$ is the Klein four group in $G / Z(G)$.

In this case we have $c s(G)=\{1,6,8,12\}$ (see $[14$, p.925]), and so by (1) we get that $\operatorname{ppcs}(G)=$ $c s(G)=\{1,6,8,12\}$, against the condition of the theorem. This completes the proof of the theorem.

We know that if $|c s(G)| \leq 3$, then $G$ is solvable (see [14, THEOREM 2.4(Ito)]). Hence, by Theorem 2.11 we obtain two corollaries. One corollary is Lemma 1.7, and another corollary is the following:

Corollary 2.12 Suppose that ppcs $(G)=$ $\{1, n, m\}$ with $n<m$ and $n / m$. Then, $G$ is one of the following types:

(1) $G$ is a quasi-Frobenius group with abelian $k$ ernel and complement;

(2) $G=P L$, where $P$ is a normal Sylow $p$-subgroup of $G$ for some prime $p$ and $L$ is an abelian p-complement of $G$, and $G / Z(G)$ is a Frobenius group. Furthermore, $Z(P)=Z(G) \cap P$ and $|c s(P)|=2$.

Note (i) Corollary 2.12 is an improvement of a part of [14, THEOREM A].

(ii) If $G$ is of type (2) in Corollary 2.12, then $\operatorname{ppcs}(G)=c s(G)=\left\{1, p^{a}, p^{b}|L / L \cap Z(G)|\right\}$ and $a>b$ (see [14, Lemma 3.3 and p.924]). So, Theorem 2.2, that is, THEOREM 1.1 of [10], is also a consequence of Corollary 2.12. 


\section{References:}

[1] B.Huppert, Character Theory Of Finite Groups, Berlin: Walter der Gruyter, 1998.

[2] M.Bianchi, D.Chilag, A.G.B.Mauri, M.Herzog and C.M.Scoppola, Applications of a graph related to conjugacy classes in finite groups, Arch. Math.58,1992, pp.126-132.

[3] Q.Kong, On an extension of Camina's theorem on conjugacy class sizes, WSEAS Transactions on Mathematics, 11, 2012, pp.898-907.

[4] E.A. Bertram, M.Herzog, A.Mann, On a graph related to conjugacy classes of groups, Bul1.London. Math.Soc. 22, 1990, pp.569-575.

[5] B.Huppert, Endlich Gruppen I. Berlin, SpringerVerlag, 1967.

[6] C.G.Shao, Q.H.Jiang, On conjugacy class sizes of primary and biprimary elements of a finite group, Sci.China.Math. 57(3), 2014, pp.491498.

[7] Q.Kong, Finite Groups with Two Class Sizes of Some Elements,Mathematical Journal of Interdisciplinary Sciences, 2, 2014, pp.191-193.

[8] D.Gorenstein, Finite Groups. New York/London: Harper \& Row, 1968.

[9] I.M.Issacs, Character Theory of Finite Groups, New York, Acadamic Press, 1976.

[10] Q.H.Jiang and C.G.Shao, FINITE GROUPS WITH THREE CONJUGACY CLASS SIZES OF CERTAIN ELEMENTS, PUBLICATION DE L'INSTITUT MATHEMATIQUE, 98(112), 2015, pp.265-270.

[11] N.BLACKBURN and B.HUPPERT, Endlich Gruppen III. Berlin-Heidelberg-New York 1982.

[12] Bo.Chen, SOME RESULTS OF LENGTH OF THE CONJUGACY CLASSES, Journal of Sichuan University (Natural Science Edition), 38, 2001, pp.176-179.

[13] J.REBMANN, F-gruppen, Arch.Math. 22, 1971, pp.225-230.

[14] S.Dolfi and E.Jabara, The structure of finite groups of conjugate renk 2,Bull London. Math. Soc. 41, 2009, pp. 916-926.

\section{Creative Commons Attribution License 4.0 (Attribution 4.0 International, CC BY 4.0)}

This article is published under the terms of the Creative Commons Attribution License 4.0

https://creativecommons.org/licenses/by/4.0/deed.en_US 